\section{Case \\ Reports}

William D.T. Kent, MD, MSc Adarsh Manjunath, BA S. Chris Malaisrie, MD
Key words: Aneurysm, dissecting/surgery; aortic aneurysm, thoracic/complications/surgery; blood vessel prosthesis implantation/methods; risk factors; stents; treatment outcome

From: Division of Cardiac Surgery, Bluhm Cardiovascular Institute, Northwestern University, Chicago, Illinois 60611

Address for reprints: S. Chris Malaisrie, MD, Galter Pavilion, Suite 11-140, 201 E. Huron St., Chicago, IL 60611

E-mail:

malaisrie@northwestern.edu

(C) 2014 by the Texas Heart ${ }^{\circledR}$ Institute, Houston

\title{
Frozen Elephant Trunk Repair
}

\author{
for Descending Thoracic Aortic Dissection \\ in a Man with a Hostile Left Pleural Cavity
}

The frozen elephant trunk procedure is a hybrid, single-staged alternative to conventional surgery for repairing diffuse pathologic conditions of the thoracic aorta. This approach is particularly advantageous in patients who have pathologic conditions of the left side of the chest, because the descending thoracic aorta can be repaired without entering a hostile pleural cavity.

We present the case of a 67-year-old man who had undergone repair of acute type A aortic dissection. He presented with aneurysmal dilation of the descending thoracic aorta secondary to chronic dissection, a large acute dissection of the proximal ascending aorta, and a large paraesophageal hernia that made him a poor candidate for conventional, 2-staged open aortic repair. We describe the hybrid frozen elephant trunk technique that we used to repair the aorta, and its broader advantages. (Tex Heart Inst J 2014;41(3):341-3)

I $\mathrm{n}$ the repair of chronic dissections that involve the ascending aorta, transverse arch, and descending thoracic aorta, a staged approach has conventionally been used. This involves replacing the ascending aorta and arch via an elephant trunk procedure through a sternotomy, followed by 2 nd-stage repair of the descending thoracic aorta through a left thoracotomy. ${ }^{1}$ In patients with a concomitant paraesophageal hernia, this strategy becomes particularly difficult because of the large sac with herniated abdominal viscera, adhesions, and possible iatrogenic gastrointestinal injury. Risks of open repair include gastric incarceration and strangulation, graft contamination, and mediastinitis. Furthermore, aortoesophageal fistula, a well-described and uniformly fatal sequela, can occur after graft replacement of the descending thoracic aorta. ${ }^{2,3}$ The frozen elephant trunk (FET) technique can reduce these risks by averting the need for thoracotomy. Through a combination of open and endovascular techniques, the FET approach enables open repair of the proximal aorta and concomitant stent-graft repair of the descending thoracic aorta, ${ }^{4}$ with no need to enter the left side of the chest in the presence of a substantial pathologic condition. We report the case of a patient with a type IV paraesophageal hernia who underwent FET repair for aneurysmal dilation of the descending thoracic aorta secondary to chronic dissection.

\section{Case Report}

In July 2012, a 67-year-old man with a history of aortic repair for acute type A dissection presented with left-sided chest pain radiating to his back. A computed tomographic (CT) angiogram showed a chronic dissection in the aortic arch and descending thoracic aorta. The dissection extended into the abdomen, with aneurysmal dilation to a maximal diameter of $6.7 \mathrm{~cm}$ in the mid descending thoracic aorta (Fig. 1). At the site of the proximal anastomosis of the previous type A dissection repair, a 1.9-cm pseudoaneurysm was found (Fig. 2). A large type IV paraesophageal hernia, containing the entire stomach and most of the transverse colon, was also identified. Fluoroscopy further delineated these findings and showed gastric organoaxial volvulus (Fig. 3). Because the images revealed evidence of interval enlargement of the descending aortic dissection, urgent intervention was indicated. After the need for an institutional review board approval was waived, we prepared to perform a single-staged FET repair.

We cannulated the right axillary artery, performed a sternotomy, cannulated the right atrium, and established cardiopulmonary bypass $(\mathrm{CPB})$. At $18^{\circ} \mathrm{F}$, hypothermic circulatory arrest was initiated; cerebral perfusion was delivered antegrade through a 


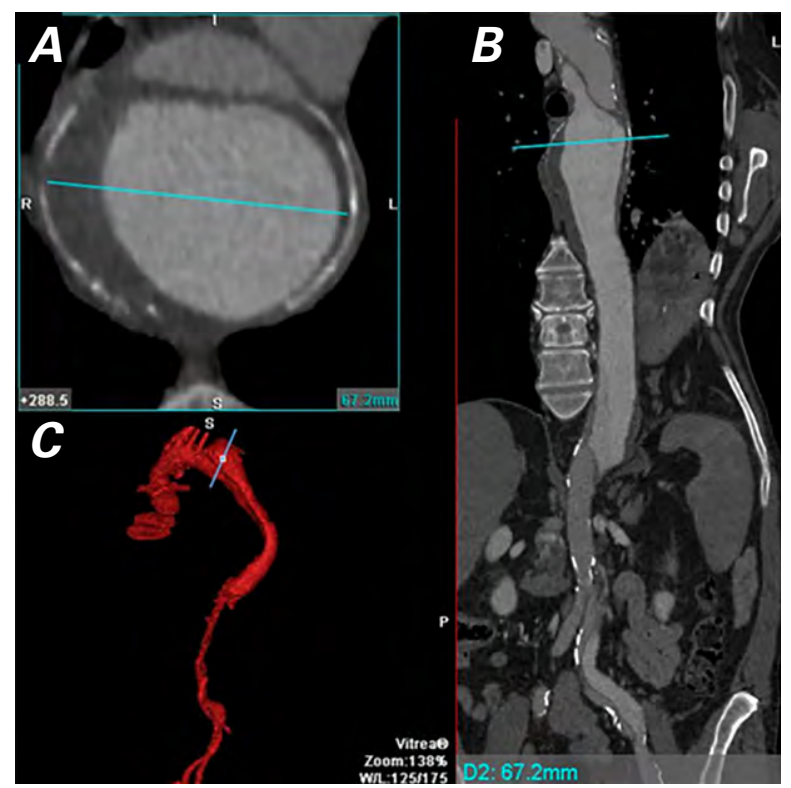

Fig. 1 Computed tomographic angiograms of a chronic descending thoracic aortic dissection show 6.7- $\mathrm{cm}$ dilation in A) axial and B) sagittal views. C) Computed tomographic angiographic reconstruction shows the morphology of the entire aorta.

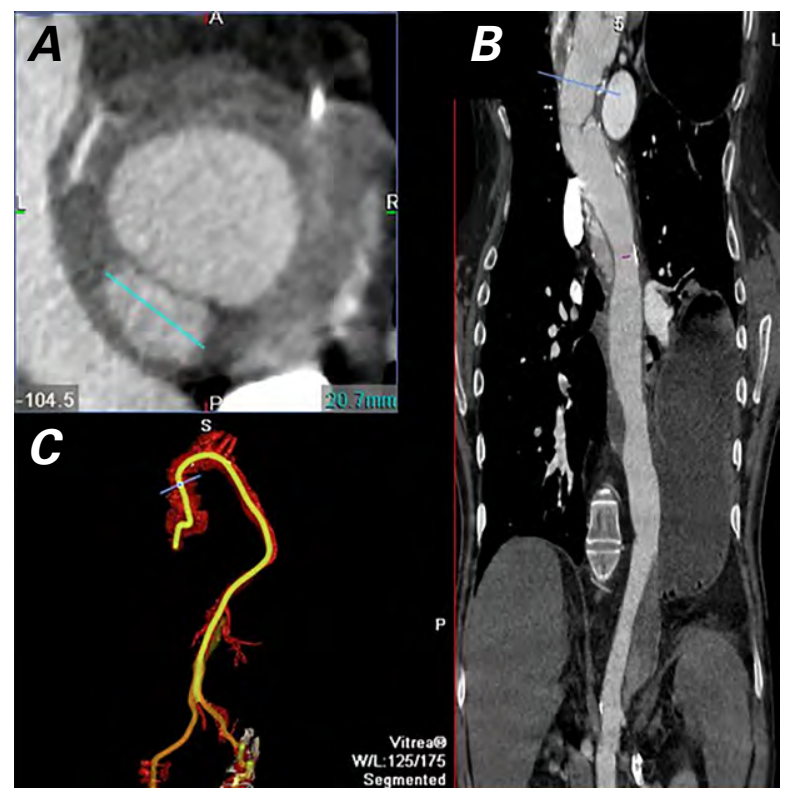

Fig. 2 Computed tomographic angiograms in $\mathbf{A}$ ) axial and B) sagittal views show a dissection of the ascending aorta after repair of a type A dissection. C) Computed tomographic angiographic reconstruction shows the dissection at the site of the proximal anastomosis.

graft on the axillary artery and a balloon-tipped catheter in the left common carotid artery. Cerebral oximetry was used for neurologic monitoring during the period of arrest. We resected the previous graft, divided the great vessels at their bases, and clamped the left subclavian artery, oversewing its stump on the arch. We transected

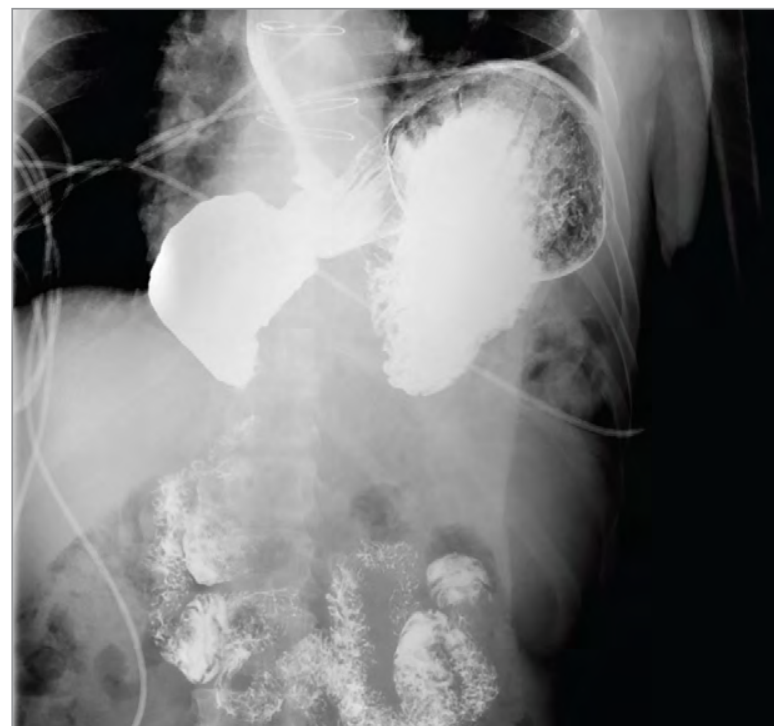

Fig. 3 Upper gastrointestinal contrast study performed with use of fluoroscopy reveals a type IV paraesophageal hernia with evidence of gastric organoaxial volvulus.

the distal arch between the left carotid and left subclavian arteries. We then placed a $24-\mathrm{mm}, 10-\mathrm{cm}$-long Vascutek $^{\circledR}$ Dacron graft (Vascutek, a Terumo company; Renfrewshire, Scotland) into the true lumen of the proximal descending thoracic aorta as an elephant trunk, and completed an anastomosis to the native aorta. We chose this approach because distal vessel runoff originated from the true lumen, and at a dimension of $30 \times 20 \mathrm{~mm}$, the true lumen was large enough to accommodate a stent-graft. We used the short elephant trunk as the proximal landing zone for endograft deployment. An Amplatz stiff wire, previously placed in the patient's left groin, was confirmed by transesophageal echocardiography to traverse the true lumen only. We retrieved the wire from the open distal arch and deployed a distal main, $26 / 26 \times 10-\mathrm{mm}$ Valiant ${ }^{\circledR}$ Thoracic Stent Graft (Medtronic Vascular; Santa Rosa, Calif) antegrade through the open chest, with its proximal landing zone in the Dacron graft. This created an FET that extended down the proximal descending thoracic aorta. We then anastomosed a 24-mm, 4-branch Vascutek $^{\circledR}$ Gelweave ${ }^{\mathrm{TM}}$ Plexus graft (Terumo) to the true lumen of the distal arch, incorporating the short elephant trunk but not the endograft. To revascularize the head vessels, we anastomosed 3 branches sequentially before re-establishing flow antegrade through the 4th branch after 52 minutes of circulatory arrest. During rewarming of the patient, we completed the proximal anastomosis with use of a 24-mm Dacron graft at the sinotubular junction, thus eliminating the dissection of the ascending aorta. After the final graft-to-graft anastomosis was performed, the aortic cross-clamp was removed and the patient was weaned from $\mathrm{CPB}$. A transesophageal echocardiogram showed stasis in the 


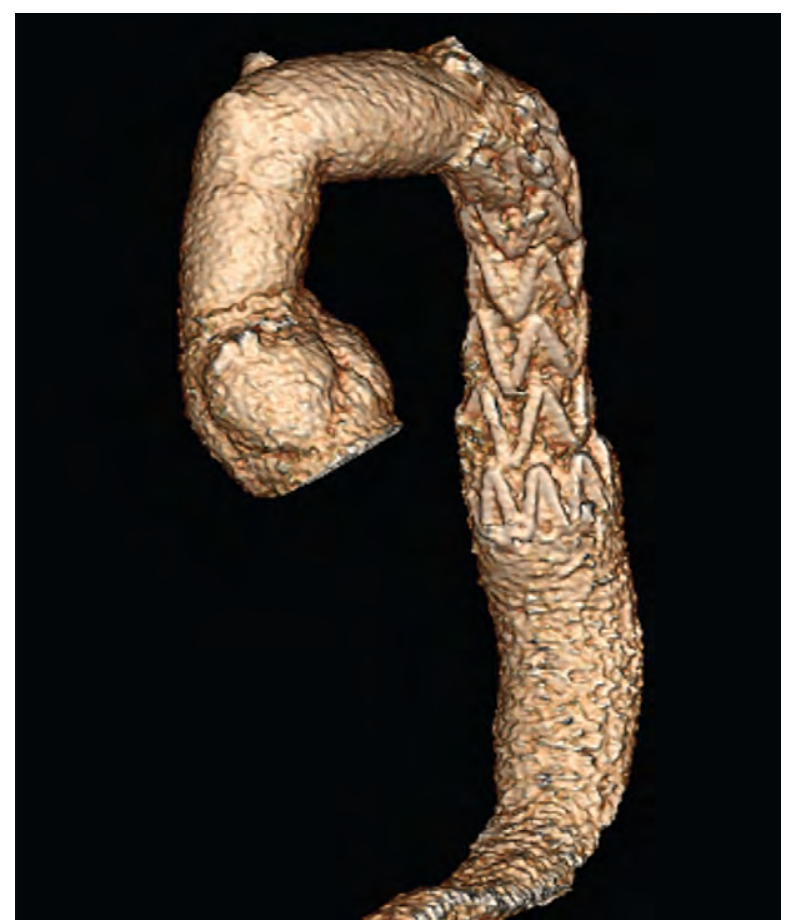

Fig. 4 Computed tomographic angiographic reconstruction shows the completed frozen elephant trunk repair and the proper positioning of the endovascular stent-graft in the descending thoracic aorta.

false lumen of the proximal descending thoracic aorta, and normal aortic valve function.

The patient's postoperative course was uneventful, and CT before his discharge from the hospital on postoperative day 7 showed a properly positioned stent-graft in the proximal descending thoracic aorta (Fig. 4) and no flow in the false lumen. The patient progressed well thereafter and returned for elective repair of his paraesophageal hernia after $2^{1 / 2}$ months. Three months later, CT showed a decrease in the maximal diameter of the descending thoracic aorta to $6.3 \mathrm{~cm}$. The patient was lost to follow-up thereafter.

\section{Discussion}

Two-staged open repair of diffuse thoracic aortic dissections has been associated with a mortality rate as high as $36 \%$. In patients with concomitant pathologic conditions of the chest, the risk of morbidity and death might be even greater during the 2nd-stage thoracotomy.

Our patient, who had a type IV paraesophageal hernia, underwent the off-label use of an endovascular stent-graft during single-staged FET repair of chronic descending thoracic aortic dissection, which was associated with a large dissection of the ascending aorta and an enlarging dissection of the descending thoracic aorta. In this case, the FET technique was a good option because it enabled endovascular repair of the descending thoracic aorta without a left thoracotomy. In addition, our use of the FET technique avoided gastrointestinal complications related to the paraesophageal hernia.

Experience with performing the FET technique is expanding, and early and midterm results have been encouraging. ${ }^{6-8}$ Although circulatory arrest is prolonged for antegrade deployment of the stent-graft, this is usually for less than 5 minutes. Advantages of the FET approach include its single stage, which eliminates the risk of death between the 2 stages of the conventional approach; less postoperative pain, because of no thoracotomy; and a comparatively shorter hospital stay. As in our patient's case, the FET procedure can be particularly advantageous in the presence of major gastroesophageal pathologic conditions, adhesions from previous chest surgery, and a history of empyema or pleurodesis. The less invasive, single-staged FET approach for treating complex aortic disease can avoid the risks associated with entering the left pleural cavity, especially when this region is hostile to intervention.

\section{References}

1. Kouchoukos NT. Frozen elephant trunk technique for extensive chronic thoracic aortic dissection: is it the final answer? Ann Thorac Surg 2011;92(5):1557-8.

2. Graham AN, McGuigan JA, Curry RC. Aortoesophageal fistula secondary to thoracic aortic aneurysm repair. J Cardiovasc Surg 1993;34(5):381-3.

3. Inoue T, Nishino T, Peng YF, Saga T. Successful one-stage operation of aortoesophageal fistula from thoracic aneurysm using a rifampicin-soaked synthetic graft. Interact Cardiovasc Thorac Surg 2008;7(2):322-4.

4. Schoenhoff FS, Schmidli J, Eckstein FS, Berdat PA, Immer FF, Carrel TP. The frozen elephant trunk: an interesting hybrid endovascular-surgical technique to treat complex pathologies of the thoracic aorta. J Vasc Surg 2007;45(3):597-9.

5. Etz CD, Plestis KA, Kari FA, Luehr M, Bodian CA, Spielvogel D, Griepp RB. Staged repair of thoracic and thoracoabdominal aortic aneurysms using the elephant trunk technique: a consecutive series of 215 first stage and 120 complete repairs. Eur J Cardiothorac Surg 2008;34(3):605-15.

6. Lima B, Roselli EE, Soltesz EG, Johnston DR, Pujara AC, Idrees J, Svensson LG. Modified and "reverse" frozen elephant trunk repairs for extensive disease and complications after stent grafting. Ann Thorac Surg 2012;93(1):103-9.

7. Murzi M, Tiwari KK, Farneti PA, Glauber M. Might type A acute dissection repair with the addition of a frozen elephant trunk improve long-term survival compared to standard repair? Interact Cardiovasc Thorac Surg 2010;11(1):98-102.

8. Baraki H, Hagl C, Khaladj N, Kallenbach K, Weidemann J, Haverich A, Karck M. The frozen elephant trunk technique for treatment of thoracic aortic aneurysms. Ann Thorac Surg 2007;83(2):S819-31. 